\title{
Nonrigid Image Registration with Subdivision Lattices: Application to Cardiac MR Image Analysis
}

\author{
R. Chandrashekara ${ }^{1}$, R. Mohiaddin ${ }^{2}$, R. Razavi ${ }^{3}$, and D. Rueckert ${ }^{1}$ \\ ${ }^{1}$ Department of Computing, Imperial College London, UK \\ ${ }^{2}$ Royal Brompton Hospital, Imperial College London, UK \\ ${ }^{3}$ Division of Imaging Sciences, King's College London, UK
}

\begin{abstract}
In this paper we present a new methodology for cardiac motion tracking in tagged MRI using nonrigid image registration based on subdivision surfaces and subdivision lattices. We use two sets of registrations to do the motion tracking. First, a set of surface registrations is used to create and initially align the subdivision model of the left ventricle with short-axis and long-axis MR images. Second, a series of volumetric registrations are used to perform the motion tracking and to reconstruct the $4 \mathrm{D}$ cardiac motion field from the tagged MR images. The motion of a point in the myocardium over time is calculated by registering the images taken during systole to the set of reference images taken at end-diastole. Registration is achieved by optimizing the positions of the vertices in the base lattice so that the mutual information of the images being registered is maximized. The presented method is validated using a cardiac motion simulator and we also present strain measurements obtained from a group of normal volunteers.
\end{abstract}

\section{Introduction}

Using magnetic resonance imaging (MRI) it is possible to obtain high resolution cine-images of cardiac motion noninvasively, enabling global functional parameters such as left ventricular volume, left ventricular mass, stroke volume, ejection fraction, and cardiac output to be measured once suitable pre-processing has been applied to the acquired images and the left ventricle (LV) has been segmented out. Although global measures of cardiac function are useful indicators of cardiac malfunction they do not reveal the regional wall motion abnormalities which are necessary for the early detection of cardiovascular diseases such as coronary heart disease. Tissue tagging ([18/2]) enables the measurement of the three-dimensional (3D) motion patterns within the walls of the heart. Radiofrequency $(\mathrm{RF})$ pulses applied at the start of the cardiac cycle produce planes of saturated magnetization in the muscle walls which appear as dark stripes when imaged immediately afterwards, and can be tracked to reconstruct dense deformation fields within the myocardium. To do this multiple stacked shortaxis (SA) and long-axis (LA) image sequences of the heart are acquired with tag planes in three mutually orthogonal directions so that the 3D motion of the heart over time can be measured. 
Numerous methods have been developed for motion field extraction using tagged MR images. Among these methods optical flow ([1476]), active contour models ([17]1]), and harmonic phase MRI (HARP) ([12]) have yielded moderately successful results although none have proved to meet all requirements in terms of accuracy and efficiency to come to dominate clinical practice. Among the main difficulties encountered is the need to estimate through-plane motion which is compounded by the effects of patient and respiratory motion. Recent work on image registration based techniques for tagged MR image analysis have yielded promising results for obtaining clinically meaningful information ([4]13 15]). The advantage of using image registration is that no assumptions are required about the nature of the tag pattern in the images. Moreover, the use of statistical measures of image similarity (e.g. mutual information) can account for the variation in tag intensity over time seen in tagged MR images. The approach in [4] uses free-form deformations (FFDs) to model the cardiac deformation. However, these models require the control points to be spaced on a regular grid covering the entire heart. A more natural approach is to adopt the control point mesh to the geometry of the LV using extended FFD models [8]. In this paper we present a new deformation model which is based on subdivision surfaces and subdivision lattices. The key advantage of this model is that the deformation of the LV can be controlled by a small number of control points and can therefore be efficiently optimized.

\section{Method}

One of the initial steps necessary for tagged MR image analysis is the segmentation of cardiac structures before subsequent motion tracking and deformation analysis. This is necessary to ensure that the blood flow patterns seen in the images do not interfere with the motion tracking of the heart muscle. One encounters a number of difficulties at this stage due to the nature of the images acquired. As there is no fully automated procedure for the accurate segmentation and fitting of surface and volumetric models to cardiac structures in tagged MR images our approach is to use a semi-automated method to construct the subdivision lattice used to model the deformation of the LV and do the motion tracking. We begin by first describing how the endocardial and epicardial surfaces of the LV are modeled using subdivision surfaces.

\subsection{Modeling the Epicardium and Endocardium with Subdivision Surfaces}

Subdivision surfaces were first proposed simultaneously by Catmull and Clark 3] and Doo and Sabin [5] in 1978 as a method for defining smooth surfaces by progressive refinement of a control polygon mesh. They are defined in terms of a base control polygon mesh and a set of geometric and topological rules which when applied to the mesh generate a sequence of polygon meshes which converge to a smooth surface. Subdivision surfaces combine the flexibility and ease of use 
of polygon meshes with arbitrary topology while retaining the smooth surface properties of spline-based surfaces.

We model the endocardial and epicardial surfaces using Catmull-Clark subdivision surfaces 3 .

Generating Subject Specific Models of the Endocardium and Epicardium Using Surface Registration. To create surface models of the endocardium and epicardium we have adopted a semi-automatic approach in which the user places point markers defining the endocardium and epicardium of the LV to which template surfaces are then registered. To aid in the placement of the markers as well as making the initial alignment of the templates we have developed a graphical user interface (GUI) First, the user places point markers delineating the endocardium on the SA and LA images. The approximate positions of the apex, base, and septum are then specified to compute a transformation matrix which makes an initial alignment of a template surface (defined using a base polygon mesh consisting of 25 vertices) with the point markers.

After this, the template subdivision surface is then deformed so that it conforms as closely as possible to the endocardial point set by minimizing the distances between the point markers and the surface. This is done by optimizing the positions of the control vertices using a gradient descent optimization procedure. The registered endocardial surface is then duplicated and used as the input surface to register to the epicardial markers. From the endocardial and epicardial surfaces we now create the volumetric model of the LV which is described in the next section.

\subsection{Construction of a Subject-Specific Model of the LV}

The idea of subdivision surfaces was extended to volumes by MacCracken and Joy 9]. Starting with a base lattice, geometrical and topological rules of subdivision are applied to generate a sequence of lattices which converge to a volume of space.

The base lattice of the LV model is constructed simply from the base polygon meshes of the endocardium and epicardium by connecting their corresponding vertices as shown in figure 1. This approach ensures that the inner and outer surfaces of the subdivision lattice correspond to the surfaces computed in section 2.1 as the geometric rules of subdivision at the boundary faces of lattices are the same as the rules of subdivision for Catmull-Clark surfaces. The base lattice contains 50 vertices.

\subsection{Motion Tracking by Image Registration}

Once the subdivision lattice is aligned with the LV we are ready to do the motion tracking. Suppose that two sets of image sequences have been acquired, a SA sequence and a LA sequence. We denote by $I_{\mathrm{SA}, t}(\mathbf{x})$ and $I_{\mathrm{LA}, t}(\mathbf{x})$ the trilinearly interpolated values of the images at position $\mathbf{x}$ and time frame $t$ in the SA and LA images respectively and we choose the images taken at the start of the cardiac 


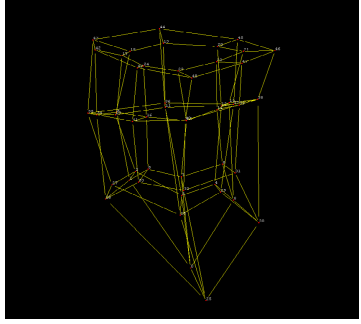

(a)

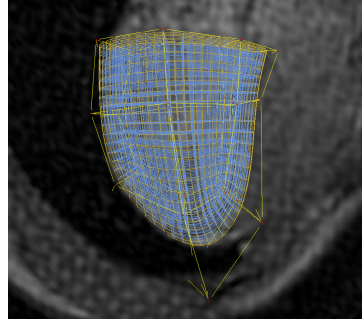

(b)

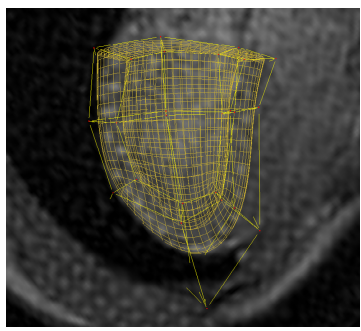

(c)

Fig. 1. This figure shows the volumetric model of the LV that is created from the surface models of the endocardium and epicardium. (a) The base lattice. (b) The model after 3 levels of subdivision. (c) The model after 3 levels of subdivision with only the boundary edges displayed.

cycle, $I_{\mathrm{SA}, 0}(\mathbf{x})$ and $I_{\mathrm{LA}, 0}(\mathbf{x})$, as the reference images (the target images). By registering the images taken during the cardiac cycle (the source images) to the target images we can determine the movement of points within the myocardium over time. Registration is being achieved by finding the displacements of the base vertices defining the subdivision lattice based transformation such that when the transformation is applied to the source images, the weighted sum of the mutual information of the images being registered is maximized (4]).

Evaluation of Similarity Measure. Before we can evaluate the degree of similarity between the images being registered we need to transform the source images into the coordinate system of the target images. Given a set of displacements of the vertices, $\mathcal{U}^{0}=\left\{\mathbf{u}_{0}^{0}, \mathbf{u}_{1}^{0}, \ldots, \mathbf{u}_{V_{0}-1}^{0}\right\}$, of the base lattice a source image is transformed into the coordinate system of the target image by finding for each voxel $\mathbf{x}_{i}$ within the subdivision lattice its transformed position, $T^{L}\left(\mathcal{U}^{0}, \mathbf{x}_{i}\right)$, in the source image. This is done by following the procedure given below,

1. We first find the cell, $\mathbf{c}_{i}^{L}$, in which the point resides. This can be done efficiently by partitioning the bounding boxes of the cells of the subdivision lattice using an octree data structure.

2. Then the local coordinates, $[u v w]^{T}$, of $\mathbf{x}_{i}$ within the cell $\mathbf{c}_{i}^{L}$ are calculated. To do this a numerical procedure based on Newton's method is used.

3. The vertices of the base lattice, $\mathcal{V}^{0}$, are now displaced by the vectors $\mathcal{U}^{0}$, and the positions of the vertices in the subdivided lattices are then updated according to the geometrical rules of subdivision given in the previous section.

4. Then, using the cell and local coordinates computed in step 2 we transform the $\mathbf{x}_{i}$ to their new positions in the deformed lattice.

The source image voxel values can now be interpolated at the transformed positions to obtain the image intensity values of the transformed source image $I_{\mathrm{SA}, t}\left(T^{L}\left(\mathcal{U}^{0}, \mathbf{x}\right)\right)$. This process is repeated for both the SA and LA images to 
obtain two transformed images, $I_{\mathrm{SA}, t}^{\prime}$ and $I_{\mathrm{LA}, t}^{\prime}$, which are then compared with their respective target images, $I_{\mathrm{SA}, 0}$ and $I_{\mathrm{LA}, 0}$. The similarity metric used is a weighted sum of the mutual information of the images being registered (note that only the image regions within the volume of the subdivision lattice are being registered). The weighting factors depend on the number of SA and LA image voxels which lie within the LV model.

Again, a gradient descent optimization procedure is used to find the optimal positions of the control points. However, other optimization methods such as the simplex method might also be suitable.

\section{Results}

\subsection{Cardiac Motion Simulator}

To validate our model we have used the cardiac motion simulator of Waks et al. 16. The motion model used in the simulator is applied to a confocal prolate spherical shell and the simulator is capable of generating realistic tagged MR images of the motion of the heart. SA and LA image sequences were generated using $k$-parameter values sampled at ten equally spaced time points between end-diastole and end-systole from figure 4 of [16]. Using the method described in section 2 the motion field within the LV volume was estimated and compared with the true motion fields obtained from the simulator. The total time taken to do the motion tracking for a sequence of images with 10 time frames was $2800 \mathrm{~s}$ on an Intel Pentium IV 3.2 GHz PC with 2 GB of RAM. For each time instant and for all voxels within the myocardium the relative error, $r$, was calculated,

$$
r=\frac{100}{N} \sum_{i=1}^{N} \frac{\left|\mathbf{t}_{i}-\mathbf{e}_{i}\right|}{\left|\mathbf{t}_{i}\right|}
$$

where $N$ is the number of voxels in the myocardium, $\mathbf{t}_{i}$ is the true displacement at voxel $i$, and $\mathbf{e}_{i}$ is the estimated displacement at voxel $i$. The relative error in the estimated displacement field was found to be $8.5 \%$.

\subsection{Normal Volunteers}

A sequence of stacked SA and LA tagged MR image sequences were also acquired from nine normal volunteers and using the method described in section 2 we derived the motion fields within the myocardium for the cardiac cycle between end-diastole and end-systole. We also derived motion fields using the FFD-based motion tracking method described in 4 so that a comparison of the two methods could be made. The myocardium at $t=0$ was then segmented along the length of the LA into three regions, the apical, mid-ventricular, and basal regions; and around the circumferential direction into the lateral, anterior, septal, and inferior regions. Figure 2 shows a comparison of the circumferential strain in the mid-ventricular region calculated using the subdivision lattice and free-form 


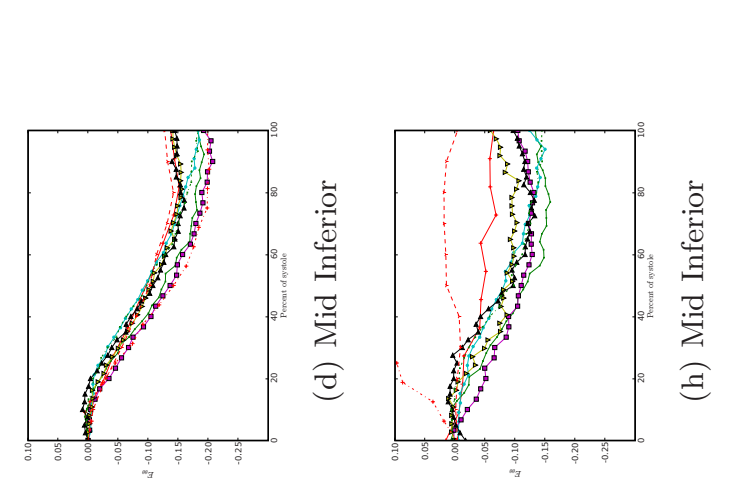

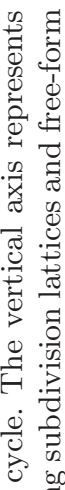

..$\overline{7}$
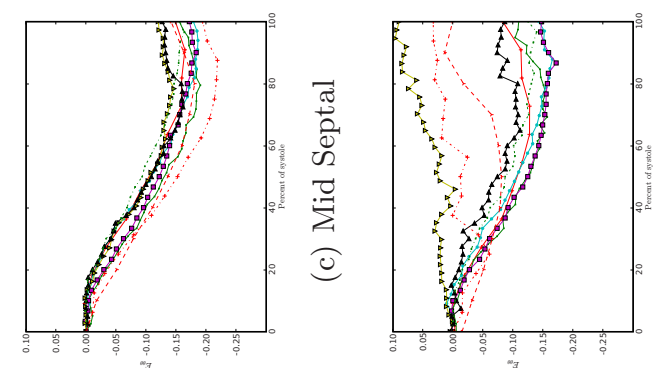

ชี

敢 击

है पु

尝 $\quad 0.07$

की

पै

है
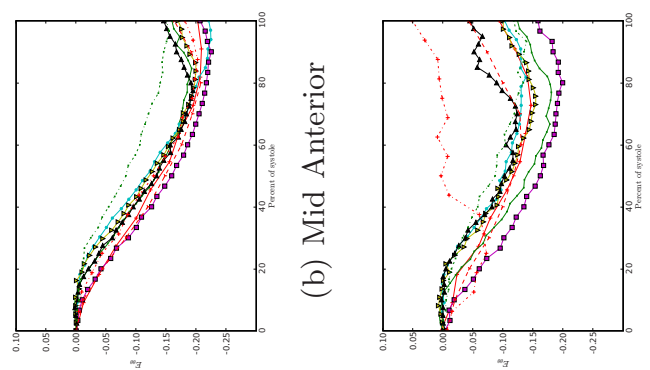

.

D

赵

त्रि

0 ?

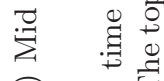

ङ

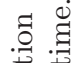

曾䓠
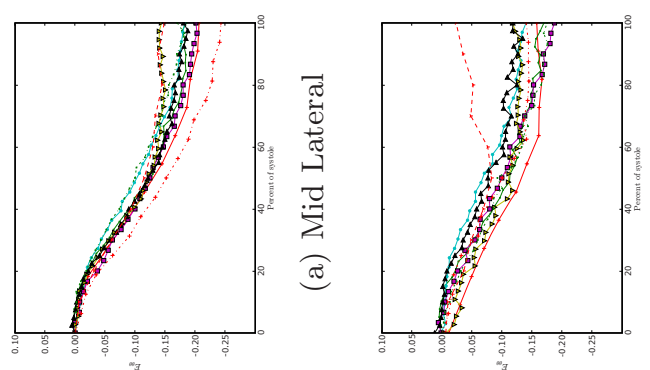

茕

สิ

疋

䟰

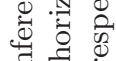

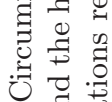

ง สี

ف․ㅠ 
deformation based motion tracking. In a normal healthy heart the circumeferential strain decreases during systole 1015. The figure indicates that the motion fields generated by the subdivision lattices are smoother than those generated by the FFDs and show less variability in the strain patterns seen across a group of normal volunteers.

\section{Discussion and Conclusions}

In this paper we have presented a method for cardiac motion tracking in tagged MR images based on nonrigid registration using subdivision lattices. We validated our method using a cardiac motion simulator and showed that accurate displacement and strain fields could be computed. The principal advantage of the method presented in this paper is the reduced number of degrees of freedom (the displacements of the base lattice vertices) which need to be optimized for. For the work presented in this paper to be of practical benefit one should build a large database of the strain distributions in normal volunteers so that comparisons could be made with the strain patterns seen in subsequent patient examinations.

\section{References}

1. Amini, A.A., Chen, Y., Elayyadi, M., Radeva, P.: Tag surface reconstruction and tracking of myocardial beads from SPAMM-MRI with parametric B-spline surfaces. IEEE Transactions on Medical Imaging 20(2), 94-103 (2001)

2. Axel, L., Dougherty, L.: Heart wall motion: Improved method of spatial modulation of magnetization for MR imaging. Radiology 172(2), 349-360 (1989)

3. Catmull, E., Clark, J.: Recursively generated B-spline surfaces on arbitrary topological meshes. Computer Aided Design 10(6), 350-355 (1978)

4. Chandrashekara, R., Mohiaddin, R.H., Rueckert, D.: Analysis of 3-D myocardial motion in tagged MR images using nonrigid image registration. IEEE Transactions on Medical Imaging 23(10), 1245-1250 (2004)

5. Doo, D., Sabin, M.A.: Behaviour of recursive subdivision surfaces near extraordinary points. Computer Aided Design 10(6), 356-360 (1978)

6. Dougherty, L., Asmuth, J.C., Blom, A.S., Axel, L., Kumar, R.: Validation of an optical flow method for tag displacement estimation. IEEE Transactions on Medical Imaging 18(4), 359-363 (1999)

7. Gupta, S.N., Prince, J.L.: On variable brightness optical flow for tagged MRI. In: Information Processing in Medical Imaging, pp. 323-334. Kluwer, Dordrecht (1995)

8. Lin, N., Duncan, J.S.: Generalized robust point matching using an extended freeform deformation model: application to cardiac images. In: Proceedings of the 2004 IEEE International Symposium on Biomedical Imaging: From Nano to Macro (2004)

9. MacCracken, R., Joy, K.I.: Free-form deformations with lattices of arbitrary topology. In: Proceedings of the 23rd Annual Conference on Computer Graphics and Interactive Techniques, pp. 181-188. ACM Press, New York (1996)

10. Moore, C.C., McVeigh, E.R., Zerhouni, E.A.: Quantitative tagged magnetic resonance imaging of the normal human left ventricle. Topics in Magnetic Resonance Imaging 11(6), 359-371 (2000) 
11. O’Dell, W.G., Moore, C.C., Hunter, W.C., Zerhouni, E.A., McVeigh, E.R.: Threedimensional myocardial deformations: Calculation with displacement field fitting to tagged MR images. Radiology 195(3), 829-835 (1995)

12. Osman, N.F., McVeigh, E.R., Prince, J.L.: Imaging heart motion using harmonic phase MRI. IEEE Transactions on Medical Imaging 19(3), 186-202 (2000)

13. Petitjean, C., Rougon, N., Preteux, F., Cluzel, P., Grenier, P.: Measuring myocardial deformation from MR data using information-theoretic nonrigid registration. In: FIMH, pp. 162-172 (2003)

14. Prince, J.L., McVeigh, E.R.: Optical flow for tagged MR images. In: Proceedings of the 1991 International Conference on Acoustics, Speech, and Signal Processing, pp. 2441-2444 (1991)

15. Tustison, N.J., Amini, A.A.: Biventricular myocardial strains via nonrigid registration of AnFigatomical NURBS models. IEEE Transactions on Medical Imaging 25(1), 94-112 (2006)

16. Waks, E., Prince, J.L., Douglas, A.S.: Cardiac motion simulator for tagged MRI. In: Proceedings of the IEEE Workshop on Mathematical Methods in Biomedical Image Analysis, pp. 182-191 (June 1996)

17. Young, A.A., Kraitchman, D.L., Dougherty, L., Axel, L.: Tracking and finite element analysis of stripe deformation in magnetic resonance tagging. IEEE Transactions on Medical Imaging 14(3), 413-421 (1995)

18. Zerhouni, E.A., Parish, D.M., Rogers, W.J., Yang, A., Shapiro, E.P.: Human heart: Tagging with MR imaging — a method for noninvasive assessment of myocardial motion. Radiology 169(1), 59-63 (1988) 